ZOOTECNIA

\title{
EVOLUÇÃO APÓS QUATRO ANOS DE APLICAÇÃO DA CAPACIDADE ANDROLÓGICA POR PONTOS (CAP) EM TOUROS NELORE E ESTUDO DA RELAÇÃO COM A PRENHEZ EM ESTAÇÃO DE ACASALAMENTO
}

\author{
EVOLUTION AFTER FOUR YEARS OF BREEDING SOUNDNESS \\ EVALUATION (BSE) IN NELLORE ZEBU BULLS AND ASSESSMENT OF \\ THE RELATIONSHIP WITH FERTILITY IN A BREEDING SEASON
}

\author{
Gustavo Eduardo Freneau ${ }^{1 *}$ \\ Jose Roberto Puoli \\ Emmanuel Arnhold ${ }^{1}$ \\ 1Escola de Veterinária e Zootecnia da Universidade Federal de Goiás, Goiânia, GO, Brasil. \\ 'Limão Consultoria Ltda. Campo Grande - MS \\ *Autor para correspondência - gfreneau@gmail.com, gfreneau@ufg.br.
}

\section{Resumo}

Estudou-se a evolução da capacidade andrológica por pontos (CAP) de 1092 touros Nelore, mantidos a pasto, durante quatro anos, e sua relação com a porcentagem de prenhez após uma estação reprodutiva. A cada ano, realizaram-se avaliações clínico-andrológicas em que mediu-se o perímetro escrotal (PE) e se coleou sêmen por eletroejaculação, para avaliação dos aspectos físicos e morfológicos dos ejaculados. A partir dos pontos obtidos pelo PE (PEP), motilidade progressiva (MOT) e morfologia espermática (MORF), computaram-se os CAP. Na estação de monta foram utilizadas 4319 fêmeas e 256 touros Nelore, em regime de pasto, para avaliação da fertilidade do rebanho. Após a escolha de 157 touros dentro das faixas A-C do CAP, foram estabelecidos, aleatoriamente, 15 grupos de touros para diferentes categorias e quantidade de fêmeas. Nos quatro anos de estudo houve aumento significativo do CAP, de 59,5 a 79,0. A PEP, MOT e MORF aumentaram no mesmo período de 17,5, 12,7 e 29,3 para 30,1, 14,8 e 34,4, respectivamente $(\mathrm{P}<0,05)$. Foram observadas correlações entre o CAP com os seus componentes, o ano e as características reprodutivas estudadas. Houve aumento significativo de touros enquadrados nas categorias A e B do CAP de $9,0 \%$ e $46,9 \%$ vs. $43,5 \%$ e $44,8 \%$, respectivamente. O CAP e a porcentagem de prenhez das fêmeas foram de $82,0 \pm 13,2$ pontos e 83,1 $\pm 8,4 \%$, respectivamente. Houve correlação $(\mathrm{P}<0,001)$ entre o CAP e a taxa de prenhez $(\mathrm{r}=0,25)$. A utilização de grupos de touros das faixas A e B do CAP favoreceu o aumento da percentagem de prenhez no rebanho. A relação touro:fêmea observada sub-utiliza o potencial reprodutivo dos touros. A técnica empregada foi importante para avaliar a fertilidade potencial e modificar as características reprodutivas da população de touros estudada.

Palavras-chave: bovinos; exame andrológico; fertilidade; morfologia espermática.

\section{Abstract}

We studied the evolution and the relationship between the fertility of a cow herd and the breeding 
soundness evaluation (BSE). During four years, 1092 Nellore zebu bulls were maintained on pasture conditions in Mato Grosso do Sul State - Brazil. After the clinic evaluation, scrotal circumference was measured and semen was collected by electro ejaculation and analyzed for physical and morphological aspects. Scores for CAP were established by sperm progressive motility (MOT), sperm morphology (MORF), and scrotal circumference (PEP). Modifications to original BSE were established by sperm concentration, sperm motility intensity, and testicles morphology. During the breeding season, 157 bulls were chosen from a-c BSE score. Fifteen reproductive groups of bulls were established randomly among three groups of females (Nellore zebu heifers, first-calf cows, mature cows, and first-calf cross-bred cows). After four years of observations, there was a significant increase in BSE varying, respectively, from 59.5 to 79.0 in 1994 to 1997 . The BSE scores PEP, MOT and MORF increased in the period from 17.5, 12.7, and 29.3 to 30.1, 14.8, and 34.4, respectively. Correlations were observed among the BSE components with the year and the studied reproductive characteristics. There was a significant increase of bulls in the BSE categories 'a' and 'b' of $9.0 \%$ and $46.9 \%$ vs. $43.5 \%$ and $44.8 \%$, respectively. The data of 1997 breeding season were: $82.0 \pm 13.2$ points and $83.1 \pm 8.4 \%$ for BSE and pregnancy rate, respectively. There was a relationship ( $\mathrm{P}<0.001)$ between BSE and pregnancy rate $(\mathrm{r}=0.25)$. The Breeding Soundness Evaluations (BSE) showed a positive correlation with the pregnancy rate in a herd with different categories of females. The use of 'a' and 'b' BSE bull groups is compatible with the high fertility potential of the herd. The ratio bull:cow used underestimates the reproductive potential of the bulls' fertility. The technique used was important to evaluate the group potential fertility and to modify the reproductive characteristics of bulls herd.

Keywords: breeding soundness evaluation; cattle; pregnancy rate; sperm morphology.

Recebido em: 29 julho 2014

Aceito em: 16 fevereiro 2016

\section{Introdução}

A capacidade andrológica por pontos (CAP) é utilizada para avaliar as condições de aptidão reprodutiva de um grupo de touros. Utilizando-se os padrões mínimos aceitáveis do CAP e uma profunda compreensão da espermatogênese e do processo de maturação espermática, é possível analisar e avaliar de forma mais eficiente os touros do rebanho ${ }^{(1)}$, além de se identificarem touros com problemas de fertilidade aparente ${ }^{(2)}$

Essa metodologia foi amplamente utilizada em estudos de reprodutores de raças europeias e zebuínas $^{(3-7)}$. Foram reportadas correlações genéticas e fenotípicas entre CAP e o crescimento corporal e testicular de touros de sobreano, calculando-se sua herdabilidade ${ }^{(8,9)}$.

Foram reportados estudos em que estes procedimentos serviram para adequar o manejo dos touros como passo prévio à tomada de decisões da estação de acasalamento ${ }^{(8)}$, para estimar parâmetros genéticos de herdabilidade em touros ${ }^{(9,10)}$ e para classificar os touros ${ }^{(11)}$. Constataram-se correlações positivas entre os componentes do CAP e a idade em touros jovens, e peso corporal, perímetro escrotal, defeitos espermáticos maiores e totais, libido e testosterona em touros adultos ${ }^{(10,12-16)}$.

Objetivou-se neste estudo avaliar o uso e a evolução do CAP em uma população de touros, bem 
como verificar a sua relação com a taxa de prenhez de rebanhos de diferentes categorias e número de fêmeas.

\section{Material e Métodos}

O trabalho foi realizado na Companhia Agrícola e Pastoril Campanário, localizada no município de Caarapó, Estado de Mato Grosso do Sul, durante quatro anos consecutivos (1994-1997). Foram avaliados 1092 touros da raça Nelore, mantidos a pasto, em regime extensivo, com várias espécies de gramíneas cultivadas, suplementação mineral e calendário profilático-sanitário de acordo com o preconizado para a região. A população de touros não tinha histórico de avaliação andrológica prévia não classificatória. Nos anos citados foram avaliados 297, 246, 293 e 256 touros, respectivamente.

Entre os meses de agosto e setembro, nos quatro anos, foram realizadas as avaliações individuais clínico-andrológica, idade, características zootécnicas, aparelho locomotor, conformação anatômica e consistência dos testículos e epidídimos. Os touros considerados aptos para essas características foram submetidos à aferição do perímetro escrotal $(\mathrm{PE})$ com auxílio de fita métrica. $\mathrm{O}$ sêmen foi coletado por meio de eletroejaculação e o ejaculado foi avaliado quanto aos aspectos físicos (aspecto, turbilhonamento, motilidade espermática individual progressiva e vigor espermático) e morfológicos, caracterizando o percentual de defeitos espermáticos maiores e menores(17).

Determinou-se a capacidade andrológica por pontos (CAP 9-105), modificado segundo Ball et al. ${ }^{(18)}$, para cada touro. O CAP individual foi composto pelas amplitudes de classes de 40-3, 40-3 e 25-3 pontos para as classes de perímetro escrotal (PEP), morfologia espermática (MORF) e motilidade individual progressiva dos espermatozóides (MOT), respectivamente. As classes de PEP para a raça Nelore foram baseadas nas faixas etárias descritas por Silva et al. ${ }^{(19)}$ com modificações de acordo com a morfologia e consistência testicular. Os touros foram distribuídos em quatro categorias de CAP, touros de CAP A (105-85), B (84-60), C (59-35) e D (<35).

Durante todos os anos, além dos touros descartados pela avaliação prévia, foram também eliminados da população touros com CAP denominados D e a maioria dos classificados com C (exceto os mais novos e com maior perímetro escrotal). No quarto ano, fez-se a avaliação clínicoandrológica de 256 animais individualmente e determinou-se o CAP. Em dezembro foi iniciada a estação de monta de 105 dias, quando 157 touros foram destinados para 4919 fêmeas. Foram utilizados somente touros com CAP A, B e, eventualmente, C. Os touros foram distribuídos, aleatoriamente, quanto à categoria e pontuação de CAP em 15 grupos ou lotes de acasalamento (G1-G15, Tabela 3). A quantidade de fêmeas Nelore usadas foi 464 novilhas, 850 primíparas, 3212 vacas e 393 primíparas mestiças (F1 de raças europeias). Foram estabelecidos 15 grupos de acasalamento com relação touro:fêmea e pontuações de CAP para os touros ao acaso. Sessenta dias após o final da estação de monta, foi realizado o diagnóstico de gestação por palpação transretal.

Avaliou-se a taxa de prenhez em função dos defeitos espermáticos menores, maiores e totais, MOT, PEP, MORF e CAP, além da relação de fêmeas por touro e a idade dos touros, por meio de modelos de regressão linear simples e da correlação de Pearson. O efeito de todas estas variáveis na prenhez 
foi avaliado de forma conjunta em um modelo misto, incluindo o tipo de fêmea (novilha, vacas, primíparas Nelore e cruzadas) como componente aleatório no modelo, e as demais variáveis (defeitos espermáticos menores, maiores e totais, MOT, PEP, MORF e CAP, além da relação de fêmeas por touro e a idade dos touros) como efeitos fixos no modelo. As variáveis em que os efeitos foram não significativos na análise de variância foram retiradas do modelo. Todas as análises foram realizadas com auxílio do software $\mathrm{R}^{(20)}$ ( $\mathrm{R}$ Core Team, 2013), utilizando-se funções do pacote lme $4^{(21)}$. Foram comparadas as médias de CAP e as frequências das faixas de CAP e comparadas por análise de variância e teste de chi quadrado, respectivamente.

\section{Resultados e Discussão}

$\mathrm{Na}$ avaliação clínica andrológica prévia, foram descartados e, consequentemente, não avaliados pelo CAP 236 touros (21,6\%), discriminados por anos da seguinte forma: 1994 - 20 touros descartados (6,3\%); 1995 - 46 descartados (17,9\%); 1996 - 39 (13,3\%); e 1997 - 33 (12,9\%). Na Tabela 1 encontram-se as características reprodutivas dos touros, nos quatro anos de observações consecutivas.

Tabela 1. Características reprodutivas de touros Nelores avaliadas pela Capacidade Andrológica por Pontos (CAP) em quatro anos consecutivos

\begin{tabular}{|c|c|c|c|c|c|}
\hline & $1^{\circ}$ ano & $2^{\circ}$ ano & $3^{\circ}$ ano & $4^{\circ}$ ano & Geral \\
\hline Idade (meses) & $72,8 \pm 25,6$ a & $67,6 \pm 30,8 a$ & $54,8 \pm 29,4 b$ & $67,9 \pm 28,1$ a & $65,7 \pm 29,2$ \\
\hline $\mathrm{PE}(\mathrm{cm})$ & $34,7 \pm 2,9 \quad b$ & $35,3 \pm 2,9 \quad b$ & $34,8 \pm 3,2 \quad$ b & $36,8 \pm 2,5$ a & $35,4 \pm 3,0$ \\
\hline PEP & $17,5 \pm 9,7 \quad \mathrm{c}$ & $23,6 \pm 9,8 \quad b$ & $24,5 \pm 9,9 \quad b$ & $30,1 \pm 10,1$ a & $23,6 \pm 11,0$ \\
\hline Motilidade (\%) & $63,2 \pm 17,9 a$ & $54,4 \pm 23,7 b$ & $63,1 \pm 17,5$ a & $57,7 \pm 18,2 b$ & $60,6 \pm 18,9$ \\
\hline Vigor $(0-5)$ & $3,4 \pm 0,7 \quad$ a & $2,9 \pm 0,9 \quad b$ & $3,4 \pm 0,8 \quad a$ & $3,4 \pm 0,7 \quad a$ & $3,2 \pm 0,6$ \\
\hline Turb. (0-5) & $1,7 \pm 0,9$ & $1,7 \pm 1,0$ & $2,0 \pm 1,1$ & $1,9 \pm 1,1$ & $1,8 \pm 1,1$ \\
\hline MOT & $12,7 \pm 6,6 \mathrm{c}$ & $13,9 \pm 7,4 \quad b$ & $16,1 \pm 6,8 \quad$ a & $14,8 \pm 6,9 \mathrm{ab}$ & $14,4 \pm 6,9$ \\
\hline Def. maiores (\%) & $16,0 \pm 19,7 \mathrm{a}$ & $12,5 \pm 8,6 \mathrm{ab}$ & $12,6 \pm 11,0 \mathrm{ab}$ & $10,1 \pm 8,7 \quad b$ & $13,1 \pm 14,1$ \\
\hline Def. totais $(\%)$ & $25,2 \pm 20,1 \mathrm{a}$ & $21,2 \pm 12,2 \mathrm{ab}$ & $17,9 \pm 11,5 b$ & $17,9 \pm 10,4 b$ & $20,6 \pm 15,1$ \\
\hline MORF & $29,0 \pm 13,6 b$ & $30,1 \pm 11,8 b$ & $30,9 \pm 11,3 \mathrm{ab}$ & $34,4 \pm 9,7 \mathrm{a}$ & $31,2 \pm 11,9$ \\
\hline CAP & $59,5 \pm 21,7 \mathrm{c}$ & $67,5 \pm 23,3 b$ & $71,6 \pm 20,9 b$ & $79,0 \pm 17,5 \mathrm{a}$ & $67,9 \pm 22,3$ \\
\hline
\end{tabular}

$\mathrm{PE}=$ perimetro escrotal; $\mathrm{PEP}=$ pontos por perimetro escrotal no CAP; Turb. $=$ turbilhonamento; $\mathrm{MOT}=$ pontos por motilidade progressiva no CAP; Def. = defeitos; $\mathrm{MORF}=$ pontos por morfologia no CAP; CAP $=$ indice de capacidade andrológica por pontos. Valores seguidos por letras distintas na linha indicam diferenças pelo teste de SNK $(\mathrm{P}<0,05)$.

Se comparados os dados do $1^{\circ}$ e $4^{\circ}$ anos houve diferença $(\mathrm{P}<0,05)$ no perímetro escrotal (PEP), na motilidade progressiva dos espermatozoides (MOT), nos defeitos maiores e totais, na morfologia 
espermática (MORF) e CAP. Estas diferenças observadas refletiram uma melhoria significativa na qualidade das características reprodutivas da população de touros no processo de avaliação periódica nesta propriedade.

A idade dos touros diminuiu, especialmente no $3^{\circ}$ ano, com a incorporação de animais de 24 meses de idade. Houve diminuição no número de touros com mais de seis anos de idade a partir do primeiro ano estudo. Eles representaram 73,4\%, 57,3\%, 35,5\% e 44,6\% nos quatro anos consecutivos (1994-1997), respectivamente. A correlação entre idade e ano foi de baixa intensidade e negativa $(\mathrm{r}=-0,11 ; \mathrm{P}<0,01)$, o que mostra uma diminuição da idade dos touros da população.

A correlação entre PE e ano foi positiva $(\mathrm{r}=0,21 \mathrm{P}<0,01)$. O PE aumentou significativamente nos touros, sendo de $2 \mathrm{~cm}$ a mais em 1997. Observaram-se coeficientes de correlação significativos $(\mathrm{P}<0,01)$ entre $\mathrm{PE}$ com motilidade progressiva dos espermatozoides (MOT), defeitos espermáticos maiores e totais, morfologia (MORF) e o índice de CAP de 0,16; 0,21; -0,20 e -0,22, 0,28 e 0,51, respectivamente. $\mathrm{O}$ aumento dos pontos do perímetro escrotal no CAP (PEP) foi de 12,6 pontos nos quatro anos, o que representa o aumento do tamanho testicular em touros de menor faixa etária e apresentou uma correlação com o ano $(\mathrm{r}=0,42 ; \mathrm{P}<0,01)$. Esta diferença em magnitude de aumento entre o PE e PEP pode ser justificada pelo descarte e ingresso no rebanho de animais mais precoces (menor idade com maior PE).

A motilidade progressiva dos espermatozoides, o vigor e o turbilhonamento apresentaram-se em valores compatíveis com os recomendados(2) (Tabela 1). Houve correlações positivas entre a motilidade progressiva com o vigor e o turbilhonamento $(\mathrm{r}=0,81$ e $\mathrm{r}=0,50 ; \mathrm{P}<0,01)$. A MOT apresentou uma elevação entre os anos com uma correlação positiva de $\mathrm{r}=0,13(\mathrm{P}<0,01)$. Os defeitos espermáticos maiores e totais tiveram uma redução significativa com o tempo e apresentaram correlações negativas com o ano $(\mathrm{r}=-0,16$ e $\mathrm{r}=-0,21 ; \mathrm{P}<0,01)$. A MORF apresentou um incremento nos quatro anos e foi observada correlação com o ano $(\mathrm{r}=0,16 ; \mathrm{P}<0,01)$. O CAP aumentou significativamente, próximo dos 20 pontos em quatro anos, sendo este correlacionado com o ano $(\mathrm{r}=0,36 ; \mathrm{P}<0,01)$. Houve elevada correlação entre o CAP e seus componentes $(\mathrm{PEP}, \mathrm{r}=$ $0,51$; MOT, $r=0,60$ e MORF, $r=0,79)$ e entre este com a motilidade progressiva $(r=0,53)$, vigor $(\mathrm{r}=0,44)$, turbilhonamento $(\mathrm{r}=0,33)$, os defeitos espermáticos totais $(\mathrm{r}=-0,67)$ e os defeitos maiores $(r=-0,61) \mathrm{P}<0,01$. Estes achados se agregam aos relatados sobre a raça Santa Gertrudis, e Nelore(5,22). Os valores de CAP apresentados em 1997 foram equivalentes aos relatados para touros de raças europeias $(8,23)$ e zebuínas(24) .

Na Tabela 2 encontram-se as frequências de categorias do CAP entre e dentro dos anos em estudo.

Houve aumento de touros nas categorias A e B do CAP ao longo do tempo, fato semelhante aos relatos que utilizaram esta metodologia como seleção de touros ao longo do tempo ${ }^{(4)}$. Possivelmente devido ao descarte de touros inadequados e à compra de touros jovens com maior perímetro escrotal, as porcentagens de touros nas categorias A e B, na avaliação do ano 1997, foram semelhantes às de estudos anteriores sobre raças taurinas ${ }^{(23,25-27)}$ e maiores que os observados por Kennedy et al. ${ }^{(28)}$ com dez grupos zootécnicos de raças europeias e seus mestiços. Neste último caso, os animais foram avaliados ao sobreano. No presente estudo, os touros foram superiores aos avaliados em clima tropical na Costa Rica $^{(29)}$, o que possivelmente se deve a diferenças raciais e de manejo. 
Tabela 2. Frequência das classes de Capacidade Andrológica por Pontos (CAP) em touros da raça Nelore, criados extensivamente em regime de pasto, em quatro anos consecutivos no Mato Grosso do Sul, Brasil

\begin{tabular}{|c|c|c|c|c|c|c|c|c|c|c|c|c|c|c|}
\hline \multirow{3}{*}{$\begin{array}{l}\text { Faixa } \\
\text { de } \\
\text { CAP }\end{array}$} & \multicolumn{3}{|c|}{$1^{\circ}$ ano } & \multicolumn{3}{|c|}{$2^{\circ}$ ano } & \multicolumn{3}{|c|}{$3^{\circ}$ ano } & \multicolumn{3}{|c|}{$4^{\circ}$ ano } & \multicolumn{2}{|c|}{ Total } \\
\hline & $\mathbf{v}$ & $\%$ & $\%$ & $n$ & $\%$ & $\%$ & $n$ & $\%$ & $\%$ & & $\%$ & $\%$ & n & \% \\
\hline & $\mathbf{N}$ & e/anos & d/ano & $\mathrm{n}$ & e/anos & d/ano & $\mathrm{n}$ & e/anos & d/ano & In & e/anos & d/ano & $\mathbf{n}$ & \\
\hline A & 25 & 11,2 & 9,0 & 16 & 7,2 & 15,7 & 83 & 37,6 & 32,7 & 97 & 43,9 & 43,5 & 221 & 25,8 \\
\hline B & 130 & 35,8 & 46,9 & 35 & 9,6 & 34,3 & 98 & 27,0 & 38,6 & 100 & 27,6 & 44,8 & 363 & 42,4 \\
\hline C & 82 & 42,5 & 29,6 & 28 & 14,5 & 27,5 & 63 & 32,6 & 24,8 & 20 & 10,4 & 9,0 & 193 & 22,6 \\
\hline D & 40 & 50,6 & 14,5 & 23 & 29,1 & 22,5 & 10 & 12,7 & 3,9 & 6 & 7,6 & 2,7 & 79 & 9,2 \\
\hline $\begin{array}{l}\text { Total- } \\
\%\end{array}$ & 277 & 32,4 & 100 & 102 & 11,9 & 100 & 254 & 29,7 & 100 & 223 & 26,0 & 100 & 856 & 100,0 \\
\hline
\end{tabular}

$\mathrm{e}^{\prime}$ anos $=$ comparaçào entre anos; d/ano $=$ dentro do ano, $\mathrm{X}^{2}(\mathrm{P}<0,05)$

Um estudo realizado com touros de raças taurinas revelou boa eficiência reprodutiva no rebanho utilizando-se relação touro:vaca de 1:44 e 1:40, sendo que não houve efeito desta relação sobre a fertilidade do rebanho ${ }^{(30)}$. A partir desta constatação, grupos de touros categorizados com CAP A e B poderiam trabalhar em estação de monta de 90 dias em uma relação touro:vaca de 1:40 sem prejudicar a fertilidade do rebanho. Pelos dados apresentados na Tabela 2, quando comparados os anos $1^{\circ}$ e $4^{\circ}$, observou-se $16,7 \%$ a menos de touros; além do fato de a propriedade apresentar um número maior de touros $\mathrm{A}$ e $\mathrm{B}$, o que permitiria acasalar $36,2 \%$ a mais de fêmeas neste último ano. Esta relação poderia ser maior, já que, nos dois anos em questão, a proporção de touros A e B foi de $9,0 \%$ e $46,9 \%$ ( $1^{\circ}$ ano) e $43,5 \%$ e $44,8 \%$ (4 $4^{\circ}$ ano), respectivamente. Outros relatos sobre as vantagens de avaliação se agregam a estes dados ${ }^{(31)}$. Este estudo confirma que o emprego sistemático da técnica do CAP em uma população de touros (permanentes e de reposição) pode modificar o seu perfil andrológico em poucos anos. A avaliação de touros (na compra e descarte) pelo CAP mostra-se como uma ferramenta básica para contribuir no incremento da eficiência reprodutiva de uma população de touros dentro do rebanho.

$\mathrm{Na}$ Tabela 3 estão representadas as características reprodutivas dos touros, a relação touro:fêmeas, a categoria e o número de fêmeas e as taxas de prenhez por grupo de touros, na estação de monta do $4^{\circ}$ ano de estudo.

Com respeito à faixa etária dos grupos de acasalamento de touros, observou-se que quando foram utilizados touros novos (G10 da Tabela 3) na relação de 1:42, o grupo apresentou uma porcentagem de prenhez elevada. A idade na população teve uma amplitude de 2-10 anos e apresentou correlações negativas com CAP e com a taxa de prenhez, respectivamente $(r=-0,60$ e $r=-0,36$; $\mathrm{P}<0,001)$. Portanto, pode-se inferir que a utilização de touros Nelore de 24-30 meses de idade é compatível com elevadas taxas de gestação em relações touro:fêmea superiores a 1:40 desde que avaliados e utilizados nas classes superior do CAP. A condição corporal dos touros não diferiu entre os grupos de touros $(\mathrm{P}>0,05)$.

$\mathrm{O}$ perímetro escrotal, como era de se esperar, foi fortemente influenciado pela idade $(\mathrm{r}=0,83$; $\mathrm{P}<0,01)$, porém, quando transformado em pontos do CAP (PEP), apresentou correlações $(\mathrm{P}<0,01)$ negativas com idade $(r=-0,70)$ e positivas com CAP $(r=0,66)$. Esta inversão de sinal na correlação da idade com o PE e PEP justifica-se porque os touros mais jovens, ingressados ao rebanho após o 
$1^{\mathrm{o}}$ ano, foram previamente escolhidos pelo maior perímetro escrotal, o que caracterizou a formação de uma população mais precoce. Esta categorização do perímetro escrotal por faixa etária é outra das vantagens da utilização do CAP, quando comparada com a sua aferição sem levar em conta a idade do touro.

Tabela 3. Características reprodutivas, frequência de touros Nelore pelo CAP, prenhez e relação touro:fêmea por grupos de touros em estação de acasalamento em Mato Grosso do Sul

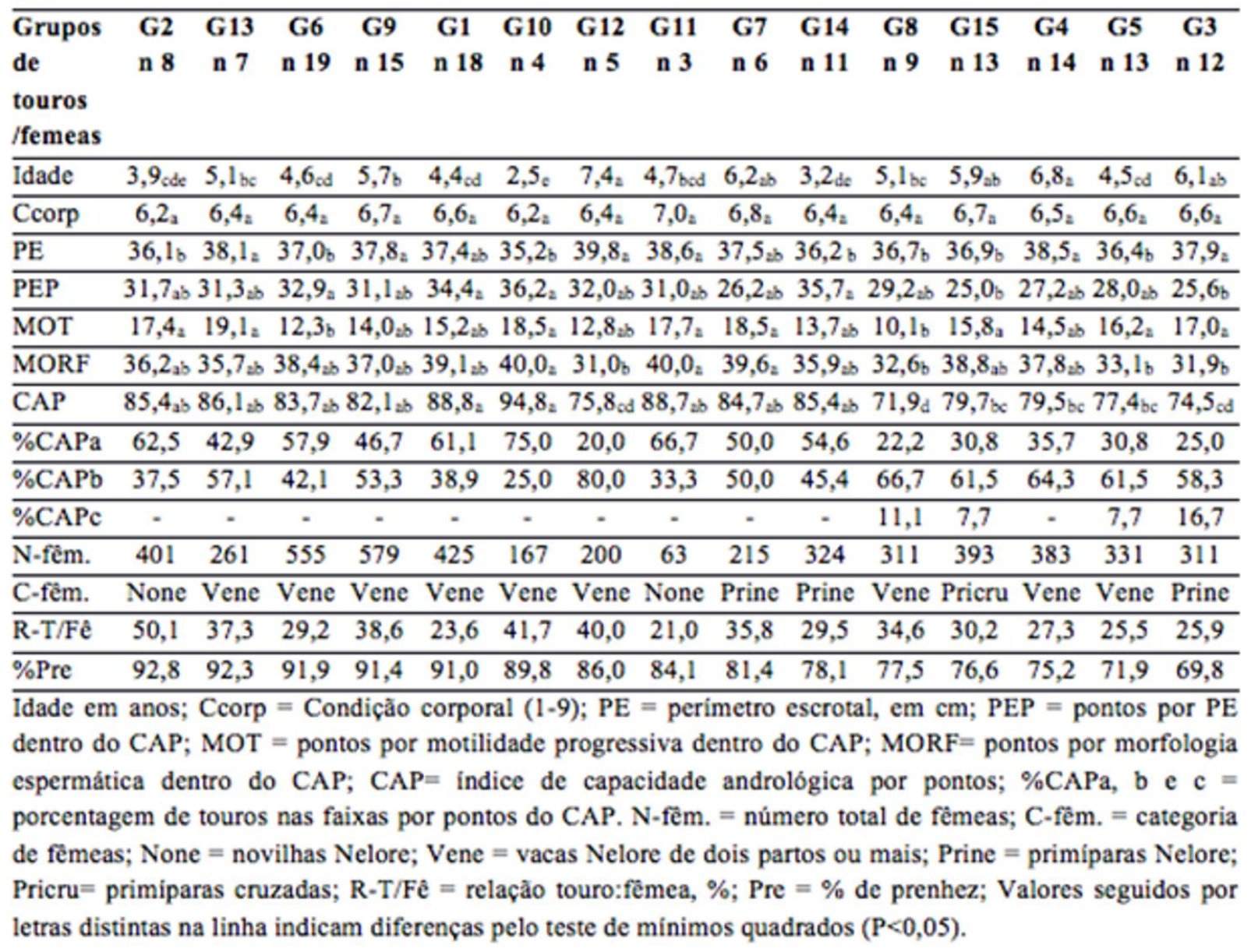

Observou-se correlação negativa entre os defeitos espermáticos totais $(\mathrm{r}=-0,16)$ e maiores $(\mathrm{r}=$ $0,22)$ com a taxa de prenhez $(\mathrm{P}<0,001)$. Esta observação coincide com trabalhos realizados em 16 fazendas no norte da Austrália, que utilizaram testes de paternidade para constatar a fertilidade de touros e observaram que, entre todos os aspectos da avaliação andrológica, a morfologia espermática foi a característica isolada que teve mais repetibilidade e relação com o números de bezerros gerados por touro ${ }^{(31)}$.

Um fato que merece atenção é a relação de touro:fêmea, que apresentou uma correlação de 0,42 $(\mathrm{P}<0,001)$. A relação existente entre a quantidade de fêmeas por touros no rebanho e a fertilidade do mesmo representa-se como uma função quadrática; assim sendo, o aumento de touros no rebanho teria uma relação positiva até um ponto de inflexão em que se voltaria negativa, com declínio da fertilidade ${ }^{(32)}$. A correlação observada no presente estudo sugere que o número de fêmeas por touro utilizado neste trabalho estaria na porção ascendente da curva. Isto permitiria especular que, em 
grupos de touros de CAP elevado, a relação de fêmeas por touro utilizada no presente rebanho foi inferior a seu verdadeiro potencial. Estes achados confirmam relatos sobre a quantidade de touros em rebanho de fêmeas ${ }^{(30)}$.

A taxa de prenhez apresentou correlação $(\mathrm{P}<0,001)$ com as seguintes características: MORF $(\mathrm{r}=$ $0,24)$, CAP $(r=0,27)$ e idade dos touros $(r=-0,21)$. Apesar de correlações de baixa intensidade, mostra que as características avaliadas nos touros apresentam relação com a prenhez. Estes achados da relação entre prenhez e CAP agregam-se aos dados apresentados por estudos sobre a mesma raça que utilizaram sincronização de $\operatorname{cios}^{(29)}$ e cios naturais com uma relação fixa de 1:25 ${ }^{(33)}$; entretanto, não se relacionam com outros estudos que não apresentaram relação com a fertilidade de rebanho ${ }^{(16)}$. Estas diferenças possivelmente se devem ao fato de esses touros terem sido alocados de forma individual ou em um grupo na relação de 1:25 touros:vacas. Como discutido acima, grupos com maior número de touros por vaca apresentariam menor eficiência reprodutiva.

Não houve correlação entre a taxa de prenhez e MOT $(r=0,004, P>0,05)$. Possivelmente, esta falta de correlação ocorreu porque esta medida de avaliação está sujeita a interferências na hora da colheita (temperatura ambiente, ventos, repouso sexual etc). Isto pode ter afetado a análise, o que demonstra a necessidade de equipamentos e medidas de manejo, para minimizar esta interferência. Outro ponto importante é que a MOT apresenta 20 pontos no CAP, o que permite que um touro com pouca pontuação neste item possa alcançar a faixa 'A' de CAP. Esta característica também foi reportada como não suficientemente boa para detectar diferencias entre os touros avaliados ${ }^{(34)}$; apesar de esse autor ter utilizado diferentes classes para motilidade progressiva dos espermatozoides. Os outros componentes do CAP (MOT e MORF) apresentaram correlações com o CAP de $r=0,43$ e $r=0,73$, ( $P<0,01)$, respectivamente, concordando com estudos anteriores ${ }^{(10,14)}$.

Os valores de CAP médio foram altos para todos os grupos e apresentaram algumas diferenças entre eles (Tabela 3). Os grupos com maiores médias de CAP apresentaram porcentagens de prenhez superiores a $80 \%$. Grupos de touros com CAP superior a 80 pontos e sem a presença de touros enquadrados na classe $\mathrm{C}$ apresentaram maiores porcentagens de prenhez. Coulter e Kozub ${ }^{(35)}$ utilizaram modelo de regressão para prever a fertilidade de grupos de touros em regime de monta e determinaram a paternidade para verificar a fertilidade individual. $\mathrm{O}$ modelo de regressão para predizer a fertilidade dos touros foi de $29 \%$ da variância total da fertilidade. Esse estudo demonstrou que seleção de touros por PE e baixa porcentagem de defeitos espermáticos maiores, somados a poucas montas orientadas com moderados números de serviços durante os testes de comportamento sexual, poderia melhorar a fertilidade de touros de corte em condições extensivas. Em última análise, até o presente momento a melhor forma de alcançar o potencial de fertilidade de um grupo de touros seria por meio da análise dos vários aspectos reprodutivos (testiculares, seminais e de comportamento sexual).

As frequências de touros dentro das classes do CAP em cada grupo de acasalamento estão apresentadas na Tabela 3. Pode-se observar que os grupos com maiores taxas de prenhez apresentaram touros A e B em proporções equilibradas. Os grupos que apresentaram touros $\mathrm{C}$ tiveram menores taxas de prenhez.

$\mathrm{Na}$ Tabela 4 encontram-se as características reprodutivas referentes aos grupos por tipo de fêmeas e a média das propriedades que integraram a estação de acasalamento.

Os valores do CAP nos touros do presente estudo foram superiores aos relatados em outros estudos com raças taurinas ${ }^{(8,22,24,25)}$, para touros taurinos adaptados aos trópicos e zebuínos ${ }^{(9,11,15,28)}$; contudo, os touros deste estudo foram selecionados de um grupo maior de touros o que não foi 
relatado nos trabalhos citados.

A diferença encontrada nas idades dos touros entre os grupos de fêmeas (Tabela 4) e a correlação observada nesta característica (CAP) com a prenhez $(\mathrm{r}=-0,26 ; \mathrm{p}<0,001)$ levam a refletir sobre a prática rotineira de manejo de se colocarem touros de maior idade nas categorias de fêmeas de menor fertilidade potencial, o que, neste estudo, não foi estabelecido.

Os valores de CAP dos touros apresentaram diferenças entre as categorias de fêmeas (Tabela 4). Da mesma forma que na análise da Tabela 3, a frequência de faixas de CAP, em cada categoria de fêmeas, mostra que a presença de touros $\mathrm{C}$ em detrimento de touros A representou em uma queda na taxa de prenhez.

Tabela 4. Características reprodutivas, frequência de touros Nelore pelo CAP, prenhez e relação touro:fêmea por categorias de fêmeas em estação de acasalamento em Mato Grosso do Sul

\begin{tabular}{lccccc}
\hline $\begin{array}{l}\text { Categoria de } \\
\text { Fêmea } \\
\text { / número de } \\
\text { touros }\end{array}$ & $\begin{array}{c}\text { Novilhas } \\
\text { Nelore / n. 11 }\end{array}$ & $\begin{array}{c}\text { Primíparas } \\
\text { Nelore / n. 29 }\end{array}$ & $\begin{array}{c}\text { Vacas } \\
\text { Nelore / n. } \\
\mathbf{1 0 4}\end{array}$ & $\begin{array}{c}\text { Primíparas } \\
\text { Cruzadas / n. }\end{array}$ & $\begin{array}{c}\text { Total } \\
\text { n. 157 }\end{array}$ \\
\hline Idade (anos) & $4,1 \pm 1,5_{\mathrm{b}}$ & $5,0 \pm 2,1_{\mathrm{ab}}$ & $5,1 \pm 1,9_{\mathrm{ab}}$ & $5,9 \pm 2,0_{\mathrm{a}}$ & $5,1 \pm 1,9$ \\
Ccorp (1-9) & $6,4 \pm 0,7_{\mathrm{a}}$ & $6,5 \pm 0,5_{\mathrm{a}}$ & $6,5 \pm 0,6_{\mathrm{a}}$ & $6,7 \pm 0,6_{\mathrm{a}}$ & $6,5 \pm 0,6$ \\
PE (cm) & $36,8 \pm 2,8_{\mathrm{a}}$ & $37,2 \pm 2,6_{\mathrm{a}}$ & $37,4 \pm 2,2_{\mathrm{a}}$ & $36,7 \pm 2,0_{\mathrm{a}}$ & $37,3 \pm 2,3$ \\
PEP & $31,5 \pm 10,2_{\mathrm{a}}$ & $29,5 \pm 10,3_{\mathrm{a}}$ & $31,2 \pm 9,3_{\mathrm{a}}$ & $25,1 \pm 11,2_{\mathrm{b}}$ & $30,4 \pm 9,8$ \\
MOT & $17,5 \pm 3,4_{\mathrm{a}}$ & $16,0 \pm 7,7_{\mathrm{a}}$ & $14,4 \pm 6,9 \mathrm{a}$ & $15,7 \pm 7,2_{\mathrm{a}}$ & $15,0 \pm 6,9$ \\
MORF & $37,3 \pm 6,0_{\mathrm{a}}$ & $35,1 \pm 8,9_{\mathrm{a}}$ & $36,6 \pm 6,9 \mathrm{a}$ & $38,8 \pm 4,1 \mathrm{a}$ & $36,6 \pm 7,1$ \\
CAP & $86,3 \pm 11,3_{\mathrm{a}}$ & $80,7 \pm 16,0_{\mathrm{b}}$ & $82,2 \pm 12,7_{\mathrm{ab}}$ & $79,7 \pm 12,7 \mathrm{~b}$ & $82,0 \pm 13,2$ \\
\% CAPa & 63,6 & 41,4 & 45,2 & 30,8 & 44,6 \\
\% CAPb & 36,4 & 51,7 & 52,9 & 61,5 & 52,2 \\
\% CAPc & - & 6,9 & 1,9 & 7,7 & 3,2 \\
N-fêm. & 464 & 850 & 3212 & 393 & 4919 \\
R-Fê/T & $42,2 \pm 13,6_{\mathrm{a}}$ & $29,3 \pm 3,7_{\mathrm{b}}$ & $30,8 \pm 6,0_{\mathrm{b}}$ & $30,2 \pm 0,0_{\mathrm{b}}$ & $31,3 \pm 6,9$ \\
\%Prenhez & $90,4 \pm 4,0_{\mathrm{a}}$ & $75,3 \pm 4,9_{\mathrm{c}}$ & $85,3 \pm 8,1_{\mathrm{b}}$ & $76,6 \pm 0,0_{\mathrm{c}}$ & $83,1 \pm 8,4$ \\
\hline
\end{tabular}

Ccorp = Condição corporal (1-9); $\mathrm{PE}=$ perimetro escrotal em $\mathrm{cm} ; \mathrm{PEP}=$ pontos por $\mathrm{PE}$ dentro do CAP; MOT = pontos por motilidade progressiva dentro do $\mathrm{CAP} ; \mathrm{MORF}=$ pontos por morfologia espermática dentro do $\mathrm{CAP} ; \mathrm{CAP}=$ indice de capacidade andrológica por pontos; \%CAPa, b e, $\mathrm{c}=$ porcentagem de touros nas faixas por pontos do $\mathrm{CAP} ; \mathrm{N}$-fêm. = número total de fêmeas; $\mathrm{R}-\mathrm{T} / \mathrm{Fe}=$ relação touro:fêmea; $\%$ Prenh $(\mathrm{obs})=\% \mathrm{de}$ prenhez observado. Valores seguidos por letras distintas na linha indicam diferenças pelo teste de Tukey $(\mathrm{P}<0,05) . \%$ Prenhez ${ }^{*} \mathrm{CAP}=$ modelo ajustado mínimos quadrados para categoria de fêmea, $\mathrm{CAP}$ e relação fêmea:touro. Valores seguidos por letras distintas na linha indicam diferenças $P<0,0001$

A relação de macho:fêmea apresentou diferenças em favor do grupo de novilhas (1:42) com elevadas taxas de prenhez e menor idade dos touros, o que sugere que touros jovens poderiam ser melhor aproveitados dentro da prática rotineira. Como era esperado, a categoria de fêmea teve uma grande influência na taxa de prenhez, o que levou à realização ajustes no modelo pelo diferente número fêmeas e categoria das mesmas. Foram observadas diferenças na taxa de prenhez entre as 
categorias de fêmeas. Como os dados provém de uma amostragem não balanceada, o modelo elaborado para análise da prenhez foi ajustado de acordo com a categoria de fêmeas, a relação macho:fêmea e o CAP dos touros.

\section{Conclusão}

De acordo com a metodologia empregada, pôde-se concluir que houve aumento significativo nos valores do CAP ao longo de quatro anos de aplicação sistemática, o que incrementa o potencial reprodutivo da população de touros na propriedade. Existem relações positivas entre o CAP e as características reprodutivas quando aplicado sistematicamente ao longo de quatro anos. A classificação dos touros pelo índice de (CAP) apresenta relação positiva com a porcentagem de prenhez em rebanho de várias categorias de fêmeas. A relação macho:fêmea utilizada rotineiramente deve ser aumentada em touros avaliados por esta técnica. A utilização de grupos de touros das classes A e B do CAP em estação de acasalamento são compatíveis com elevada fertilidade no rebanho.

\section{Agradecimentos}

Os autores agradecem aos responsáveis da Companhia Agrícola e Pastoril Campanário - Carapoó MS pelos dados fornecidos para realizar este trabalho.

\section{Referências}

1. Alexander JH. Bull breeding soundness evaluation: A practitioner's perspective Theriogenology, 2008, 70:469-72.

2. Kastelic JP, Thundathil JC Breeding Soundness Evaluation and Semen Analysis for Predicting Bull Fertility. Reprod Dom Anim, 2008; 43, Suppl. 2:368-373, 2008.

3. Barth AD, Cheryl L, Waldner FD. Factors affecting breeding soundness classification of beef bulls examined at the Western College of Veterinary Medicine. Can Vet J, 2002; 43:274-28.

4. Smith MF, Morris DL, Amoss MS, Parish NR, Williams JD, Wiltbank JN. Relationships among fertility, scrotal circunference, seminal quality and libido in Santa Gertrudis bulls. Theriogenology, 1981; 16(4):379397.

5. Godfrey RW; Randel RD, Parish NR. The effects of using the Breeding soundness evaluation as a selection criterion for Santa Gertrudis bulls on bulls subsequent generations. Theriogenology 1988; 30(6):1059-68.

6. Chenoweth PJ, Chase Jr CC, Thatcher MJD, Wilcox CJ, Larsen RE. Breed and other effects on reproductive traits and breeding soundness categorization in young bulls in Florida. Theriogenology, 1996; 46(7):1159-70.

7. Fordyce G, Entwistl, K, Norman S; Perry V, Gardiner B, Fordyce P. Standardizing bull breeding soundness evaluations and reporting in Australia. Theriogenology,2006; 66:1140-48. 
8. Smith BA, Brinks JS, Richardson GV. Estimation of genetic parameters among breeding soundness examination components and growth traits in yearling bulls. J.Anim.Sci., 1989; 67(11): 2892-96

9. Dias JC, Andrade VJ, Martins JAM, Emerick LL, Vale Filho VR. Correlações genéticas e fenotípicas entre características reprodutivas e produtivas de touros da raça Nelore. Pesq.Agropec.Bras.,2008; 43(1):5359.

10. Vale Filho VR, Bergman JAG, Andrade VJ, Quirino CR, Reis SR, Mendoça RMA. Caracterização andrológica de touros Nelore, selecionados para primeira estação de monta. Rev.Bras.Repro.Anim. 1997; 21(2):42-45

11. Vale Filho VR, Andrade VJ, Quirino CR, Graça DS, Bergman JAG, Salvador DF, Ribeiro Filho, AL, Reis SR. Perfil andrológico de touros Tabapuã (Bos teurus indicus) de um e dois anos de idade, criados extensivamente nos estados de minas Gerais Bahia e Espírito Santo, Brasil. Rev.Bras.Repro.Anim. 2001; 25(2):189-192.

12. Godfrey RW, Dodson RE. Breeding soundness evaluations of Senepol bulls in the US Virgin Islands. Theriogenology, 2005; 63:831-40.

13. Dias JC, Andrade VJ, Emerick LL, Martins JAM, Vale Filho VR, Silva MA. Teste da libido em touros jovens guzerá e suas associações com características reprodutivas e níveis séricos de testosterona Archives of Veterinary Science, 2009; 14(4):.204-213.

14. Corrêa AB, Vale Filho VR, Corrêa GSS, Andrade VJ, Silva MA, Dias JC. Características do sêmen e maturidade sexual de touros jovens da raça Tabapuã (Bos taurus indicus) em diferentes manejos alimentares. Arq. Bras. Med. Vet. Zootec, 2006; 58(5):823-30.

15. Folhadella IM., Sá WF, Ferreira AM., Camargo LSA. Viana JHM, Ramos AA, Silva MVGB. Características andrológicas de touros da raça Gir. Arq. Bras. Med. Vet. Zootec., 2006; 58(5):809-815.

16. Lopes FG, Guimarães JD, Costa EP, Carvalho GR, Neto TM. Avaliação andrológica por pontos e comportamento sexual em touros da raça Nelore. R. Bras. Zootec., 2009; 38(6):1018-25.

17. Blom, E. The ultrastructure of some characteristics sperm defects and a proposal for a new classification on the bull spermiogram. Nord. Vet. Med., , 1973; 25(7-8):383-391.

18. Ball L, Ott RS, Mortimer RG, Simons JC. Manual for breeding soundness examination of bulls. J.Soc.Theriogenology, 1983; 12:1-65.

19. Silva, AEDF, Dode MAN, Unanian MM. Capacidade reprodutiva do touro de corte: Funções, anormalidades e outros fatores que a influenciam. EMBRAPA-CNPGC.1993, Doc.51, 128p,

20. R Core Team (2013). R: A language and environment for statistical computing. R Foundation for Statistical Computing, Vienna, Austria. URL http://www.R-project.org/. Acessado em 21 de abril de 2013.

21. Bates, D, Maechler, M; Bolker, B. (2013). lme4: Linear mixed-effects models using S4 classes. R package version 0.999999-2. http://CRAN.R-project.org/package=lme4. Acessado em 21 de abril de 2013.

22. Lopes, F. G.; Guimarães, J. D.; Costa, E. P.; Carvalho, G. R.; Neto, T. M. Avaliação andrológicapor pontos e comportamento sexual em touros da raça Nelore. Rev.Bras.Zoot. 2009, 38(6):1018-25.

23. Bruner KA, Mccraw RL, Whitacre MD,Van-Camp SD. Breeding soundness examination of 1,952 yearling beef bulls in North Carolina. Theriog.enology, 1995; 44(1):129-145.

24. Lopes F G; Koetz Jr C; Barca Jr F A; Okano, W; Da Silva L C; Da Silva Jr. M A G Maturidade sexual e classificação andrológica por pontos (Cap) em touros jovens da raça Nelore puros de origem (Po). Biosci. J., 2013, 29(1):168-173 
25. Chenoweth PJ, Farin PW, Mateos ER, Rupp GP, Pexton JE. Relationship between Breeding Soundness and sex drive classification in beef bulls. Theriogenology, 1988; 30(2):227-233.

26. Spitzer JC, Hopkins FM, Webster HW, Kirkpatrick FD, Hill HS. Breeding soundness examination of yearling beef bulls. J.A.V.M.A., 1988; 193(9):1075-79.

27. Higdon III HL, Spitzer JC, Hopkins FM, Bridges Jr, WC. Outcomes of Breeding soundness evaluations of 2898 yearling bulls subjected to different classification systems. Theriogenology, 2000; 53:1321-32.

28. Kennedy SP, Spitzer JC, Hopkins FM, Higdon Iii HL, Bridges Jr WC. Breeding soundness evaluations of 3648 yearling beef bulls using the 1993 Society for Theriogenology guidelines. Theriogenology, 2002; 58: 947-61

29. Chacón J, Pérez E, Müller E, Söderquist L, Rodríguez Martínez, H. Breeding soundness evaluation of extensively managed bulls in Costa Rica. . Theriogenology, 1999; 52:221-231.

30. Rupp GP, Ball L, Shoop MC. et al. Reproductive efficiency of bulls in natural service: Effects of male to female ratio and single versus multiple sire breeding groups. J.Am.Vet.Ass. 1977; 171:639.

31. Guimarães, J. D; Guimarães, S. E. F.; Siqueira, J. B.; Pinho R. O.; Eler, J. P.; Ferraz, J. B. S.; Silva, M. R.; Borges, J. C. Seleção e manejo reprodutivo de touros zebu. R. Bras. Zootec., 2011, 40:379-388.

32. Fitzpatrick LA, Fordyce G, Mcgowan MR, Bertram JD, Doogan VJ, De Faverif J, Miller, J, Holroyd RG. Bull selection and use in northern Australia Part 2. Semen traits. Anim.Reprod.Sci, 2002; 71:39-49

33. Salisbury GW, Vandemark NL, Lodge JR. Fisiologia de la reproducción e inseminación artificial de los bóvidos. ed Acribia, Zaragoza, 831 p., 1978.

34. Chaves, R. M.; Souza, J. A. T. Nascimento, I.M.R.; Lopes J.B.; Pontes C. B.; Bezerra F.Q.G; Machado, P. P.; Santos, M.H.B. Avaliação da capacidade reprodutiva de touros da raça Nelore através da classificação andrológica por pontos (CAP) e do teste da libido. Medicina Veterinária, 2007, 1(1):26-32.

35. Garcia-Paloma J A. A bull breeding soundness evaluation system with greater emphasis on scrotal circumference. Pesq. Vet. Bras. 2015, 35(9):817-821.

36. Coulter GH, Kozub GC. Efficacy of methods to test fertility of beef bulls used for multiple-sires breeding under range conditions. J.Anim.Sci, 1989; 67:1757. 